05

\title{
Теоретическое построение и расчетно-экспериментальное подтверждение условия выбора материала антидиффузионного слоя терможаростойкого биметаллического провода
}

\author{
(C) И.В. Витковский, ${ }^{1}$ С.Н. Ромашин, ${ }^{2}$ В.С. Федеряева, ${ }^{1, \uparrow}$ В.С. Шоркин ${ }^{2}$ \\ ${ }^{1}$ Акционерное общество „НИИЭФА им. Д.В. Ефремова“, \\ 196641 Санкт-Петербург, Россия \\ ${ }^{2}$ Орловский Государственный Университет им. И.С. Тургенева, \\ 302026 Орел, Россия \\ ฯ e-mail: federyaeva_v.s@sintez.niiefa.spb.su, vshorkin@yandex.ru
}

(Поступило в Редакцию 5 апреля 2017 г.)

Приведено теоретическое обоснование и экспериментальное подтверждение условия выбора материала антидиффузионных слоев терможаростойкого биметаллического обмоточного провода электромагнитных насосов и электродвигателей. Теоретическое обоснование сформулировано на основании представления об отсутствии адгезии между элементами конструкции провода, а следовательно, и взаимной диффузии материалов его составляющих. Для построения количественного условия выбора материала антидиффузионного слоя биметаллического провода принята модель сплошного упругого твердого тела.

DOI: 10.21883/JTF.2018.10.46497.2287

\section{Введение}

На современном этапе развития атомной промышленности и машиностроения предъявляются все более высокие эксплуатационные требования к конструкционным и электротехническим материалам магнитогидродинамических машин и электродвигателей. Особое значение приобретает возможность их эксплуатации при температурах, достигающих $400-700^{\circ} \mathrm{C}[1]$.

Терможаростойкий обмоточный провод, предназначенный для эксплуатации в условиях воздействия высоких температур, представляет собой многослойную систему, внутренний слой которой выполнен из меди или хромниобиевой бронзы, заключенной в оболочку из коррозионно-стойкой стали аустенитного класса, покрытой изоляцией — армированными стеклонитью фосфатами.

Отметим, что качество терможаростойких проводов обеспечивается высокой технологической проработкой и глубоким пониманием процессов адгезии и диффузии. В свою очередь, технология изготовления должна базироваться только на высококачественных исходных материалах. При формировании такого провода необходимо обеспечить отсутствие взаимодиффузии материалов токопроводящей жилы (проводника), обечайки, изоляции, поскольку взаимное проникновение контактирующих материалов с течением времени приводит к существенному изменению физико-химических и электрических характеристик токопроводящей жилы и изоляции.

Кроме того, с ростом температуры ускоряются процессы как окисления изоляции и защитной оболочки, так и взаимодиффузии продуктов окисления и материалов токопроводящей жилы.
Перечисленные выше процессы оказывают влияние на срок службы таких обмоточных проводов, а следовательно, и электрических машин. При этом нетрудно заметить, что движущей силой процессов нарушения исходной физико-химической структуры провода является диффузия. Значит, ее ослабление или полная ликвидация должны способствовать увеличению срока службы обмоточного провода. Существенно ослабить процесс взаимной диффузии контактирующих материалов можно путем исключения их адгезии [2].

Действительно, материал реального твердого тела $B_{(1)}$ всегда имеет дефекты структуры, например, в виде вакансий. При этом энергия случайных колебаний отдельных частиц может превысить значение энергии активации $Q_{a}[2,3]$ и они, становясь делокализованными [3], совершают элементарный акт самодиффузии скачок из одного положения в другое. В каждый момент времени материал содержит определенное число $N_{d}$ делокализованных частиц, некоторая часть которых достигает граничной поверхности (например, поверхности $A_{(1)}$ медной жилы $B_{(1)}$ или $A_{(2)}$ стальной оболочки).

Для совершения акта сублимации частицам с поверхности $A_{(n)}(n=1,2)$ необходимо обладать энергией, превышающей энергию сублимации $Q_{s}$. Поскольку $Q_{s}>Q_{a}$ (например [4,5], при температуре $T=293 \mathrm{~K}$ у меди (токопроводящая жила): $Q_{s}=337 \mathrm{~kJ} / \mathrm{mol}, Q_{s}>Q_{a}, \mathrm{y}$ железа (стальная защитная оболочка): $Q_{s}=391 \mathrm{~kJ} / \mathrm{mol}$, $Q_{a}=270 \mathrm{~kJ} / \mathrm{mol}$ ) преодолеть барьер $Q_{s}$ может лишь часть $N_{d}$ сублимировавших $N_{s}$ частиц. При наличии адгезии для совершения элементарного акта диффузии частица должна обладать энергией активации, характерной для межрешеточной переходной фазы, появляющейся из-за возникновения связей между атомами решеток $[2,6,7]$. Эта энергия должна быть соизмеримой 
с энергиями активации самодиффузии (или меньшей их) и меньшей энергии сублимации.

Для минимизации числа сублимировавших частиц, достигших поверхности $A_{(p)}$ защищаемого тела $B_{(p)}$, необходимо затруднить их переход из $B_{(n)}$, в $B_{(p)}$. Для этого зазор между граничными поверхностями $A_{(n)}$ и $A_{(p)}$ тел $B_{(n)}$ и $B_{(p)}$ предлагается заполнить антиадгезионным материалом $B_{(3)}$. Таким образом, слой материала между телами $B_{(1)}$ и $B_{(2)}$, защищающий от взаимной диффузии их материалов (защитный, антидиффузионный слой), является одновременно и антиадгезионным.

Цель работы состоит в теоретическом построении условий, которым должны удовлетворять свойства материала защитного слоя по отношению к свойствам материалов защищаемых тел $B_{(1)}$ и $B_{(2)}$ и которые могут служить основанием выбора соответствующего материала.

\section{Основные теоретические положения и допущения}

Адгезионное взаимодействие связано с проявлением сил Кулона и Ван-дер-Ваальса при межчастичных взаимодействиях [8-11]. Учитывая межчастичный характер взаимодействия твердых тел, его потенциальную энергию можно представить в виде суммы потенциальных энергий взаимодействий всех пар, троек и т.д. частиц, составляющих эти тела [12-14]. Если тройные взаимодействия имеют флуктуационную природу $[11,15,16]$, то их можно описать, используя групповые разложения, с помощью функций Майера для парных взаимодействий [17]. Допустима ситуация $[11,18]$, когда силы действия одного тела на другое, порожденные парными и тройными взаимодействиями, имеют разнонаправленный характер и преобладают силы отталкивания контактирующих тел. На основании сказанного полагаем, что материал защитного слоя должен отталкиваться токопроводящей жилой и ее защитной оболочкой.

Для построения количественного условия выбора материала антидиффузионного слоя для биметаллического провода принята модель сплошного упругого твердого тела, основанная на следующих допущениях:

1. Материалы взаимодействующих тел $B_{(n)}(n=$ $=1,2,3-$ их номера) считаются сплошными. В качестве отсчетного для них принимается термодинамическое состояние материалов вдали от свободных границ или поверхностей контакта. Их плотности $\rho_{(n)}$ и температуры $T_{(n)}$ распределены равномерно и с течением времени не меняются. При этом $T_{(1)}=T_{(2)}=T_{(3)}$.

2. Контактирующие между собой пары тел: $\left(B_{(1)}, B_{(3)}\right.$, $\left.B_{(2)}, B_{(3)}\right),\left(B_{(1)}, B_{(2)}\right)$ или $\left(B_{(1)}, B_{(2)}\right)$ взаимодействуют между собой с помощью потенциальных сил, моделирующих силы Ван-дер-Ваальса [19-21]. Разделенные промежуточным слоем $B_{(3)}$ тела $B_{(1)}$ и $B_{(2)}$ не взаимодействуют.
3. Рассматриваемые тела считаются состоящими из упорядоченных пар $\left(d B_{1(n)}, d B_{2(p)}\right)$ и троек $\left(d B_{1(n)}\right.$, $\left.d B_{2(p)}, d B_{3(q)}\right)(n, p, q$ - номера тел, которым принадлежат частицы; при совпадении номеров в скобках частицы из одного тела) взаимодействующих между собой частиц. Положение любой частицы $d B_{2(p)}$ относительно частицы $d B_{1(n)} \equiv d B_{(n)}$ при деформировании материала меняется и в текущем состоянии определяется радиусом-вектором $\mathbf{L}_{1(n) 2(p)}=\mathbf{R}_{2}(p)-\mathbf{R}_{1}(n)$ с длиной $L_{1(n) 2(p)}=\left|\mathbf{L}_{1(n) 2(p)}\right|$. Здесь $\mathbf{R}_{i(j)}, \mathbf{r}_{i(j)}-$ радиусы-векторы положения частицы $d B_{i(j)}$ соответственно в текущем и отсчетном состоянии материала; $\mathbf{u}_{i(j)}$ - перемещение частицы $d B_{i(j)}$ из положения $\mathbf{r}_{i(j)}$ в положение $\mathbf{R}_{i(j)}, i=2,3 ; j=p, q ; \Delta \mathbf{u}_{1(n) 2(p)}=$ $=\mathbf{u}_{2(p)}-\mathbf{u}_{1(n)}-$ относительное смещение частиц при переходе из отсчетного состояния в текущее. В отсчетном состоянии $\mathbf{L}_{1(n) 2(p)}=\mathbf{I}_{1(n) 2(p)}$, в текущем состоянии: $\mathbf{L}_{1(n) 2(p)}=\mathbf{I}_{1(n) 2(p)}+\delta \mathbf{u}_{1(n) 2(p)}$.

Смещение $\delta \mathbf{u}_{1(n) 2(p)}$ представлено в виде ряда по внешним степеням $\mathbf{I}_{1(n) 2(p)}^{m}$ вектора $\mathbf{I}_{1(n) 2(p)}, m=0,1, \ldots, \infty$. Его коэффициентами являются тензоры $\left((-1)^{m} / m !\right) \nabla^{m} \mathbf{u}$. Здесь $\nabla=d(\ldots) / d \mathbf{r}_{1(n)}$ - дифференциальный оператор Гамильтона, для него справедливо: $d(\ldots) / d \mathbf{r}_{1(n)}=$ $=-d(\ldots) / d \mathbf{I}_{1(n) i(j)} ; \mathbf{u} \equiv \mathbf{u}_{1(n)}$. В расчетах используются первые два члена ряда. Тоже самое можно сказать о положении частиц $d B_{3(q)}$ относительно частиц $d B_{1(n)} \equiv$ $\equiv B_{(n)}$. Положение $d B_{3(q)}$ относительно $d B_{2(p)}$ определяется вектором $\mathbf{L}_{2(p) 3(q)}=\mathbf{L}_{1(n) 3(q)}=\mathbf{L}_{1(n) 2(q)}$.

4. По аналогии с [12-14] допускается, что полная потенциальная энергия каждого из рассматриваемых тел и любой их пары равна сумме потенциальных энергий $d U_{1(n) 2(p)}^{(2)}=\Phi_{1(n) 2(p)}^{(2)} d V_{1(n)} d V_{2(p)}, d U_{1(n) 2(p)}^{(3)}=$ $=\Phi_{1(n) 2(p) 3(q)}^{(3)} d V_{1(n)} d V_{2(p)} d V_{3(q)}$ взаимодействия частиц с объемами $d V_{1(n)}, d V_{2(p)}$ и $d V_{3(q)}$, входящих соответственно в пары $\left(d B_{1(n)}, d B_{2(p)}\right)$ и тройки $\left(d B_{1(n)}, d B_{2(p)}\right.$, $\left.d B_{3(q)}\right)$. Коэффициенты $\Phi_{1(n) 2(p)}^{(2)}$ и $\Phi_{1(k) 2(p) 3(q)}^{(3)}-$ это потенциалы, которые для однородного изотропного материала зависят только от расстояний между частицами (здесь и далее верхний индекс в скобках означает число взаимодействующих частиц).

$$
\begin{gathered}
\Phi_{1(n) 2(p)}^{(2)}=\Phi_{0(n p)}^{(2)} \Psi_{1(n) 2(p)}, \\
\Phi_{1(n) 2(p) 3(q)}^{(3)}=\Phi_{0(n p q)}^{(3)} \Psi_{1(n) 2(p)} \Psi_{2(p) 3(q)} \Psi_{3(q) 1(n)} .
\end{gathered}
$$

В выражениях (1) и (2) $\Psi_{i(j) g(s)}=\Psi_{i(j) g(s)}\left(\eta_{i(j) g(s)}\right)-$ безразмерные функции безразмерного аргумента $\eta_{i(j) g(s)}=\beta_{j s} L_{i(j) g(s)}$, описывающие зависимость парного потенциала взаимодействия от расстояния между взаимодействующими частицами. В отсчетном состоянии взаимодействующих материалов, когда $L_{i(j) g(s)}=$ $=l_{i(j) g(s)}$, вид зависимости $\Psi_{i(j) g(s)}\left(\eta_{i(j) g(s)}\right)$ сохраняется. Эта возрастающая зависимость, $\Psi_{i(j) g(s)}(0)=-1$, при $\eta_{i(j) g(s)} \rightarrow \infty: \Psi_{i(j) g(s)} \rightarrow 0$. Параметры $\beta_{j s}, \Phi_{0(n p)}^{(2)}$ и $\Phi_{0(n p q)}^{(3)}$ характеризуют свойства взаимодействующих 
материалов и нуждаются в теоретическом или экспериментальном определении. В работе принято:

$$
\Phi_{i(j) g(s)}=\exp \left(-2 \eta_{i(j) g(s)}\right)-2 \exp \left(-\eta_{i(j) g(s)}\right) .
$$

5. Энергия $d W_{(n)}\left(\mathbf{R}_{(\mathbf{n})}\right)=w_{(n)}\left(\mathbf{R}_{(n)}\right) d V_{(n)}$ бесконечно малой частицы $d B_{(n)}$ тела $B_{(n)}$, взаимодействующего с телом $B_{(p)}(p \neq n)$, представлена в виде

$$
\begin{aligned}
& w_{(n)} d V_{(n)}=\left(w_{(n n)}+w_{(n p)}\right) d V_{(n)} \\
& =\left[\int_{V(n)} \Phi_{(n n)}^{(2)} d V_{(n)}+\frac{1}{2 !} \int_{V(n)} \int_{V(n)} \Phi_{(n n n)}^{(3)} d V_{(n)} d V_{(n)}\right] d V_{(n)} \\
& +\left[\int_{V(p)} \Phi_{(n p)}^{(2)} d V_{(p)}+\frac{1}{2 !} \sum_{\alpha=n, p_{V(p)}} \int_{V(n)} \Phi_{(n p \alpha)}^{(3)} d V_{(\alpha)}\right] d V_{(n)} .
\end{aligned}
$$

Здесь $w_{n n}$ - объемная плотность потенциальной энергии, возникшей при взаимодействии частиц тела $B_{(n)}$ между собой; $w_{n p}$ - добавка, возникшая из-за взаимодействия тела $B_{(n)}$ с телом $B_{(p)}$.

Сила $d \mathbf{F}_{(n)}=\mathbf{f}_{(n)} d V_{(n)}$, действующая на частицу $d B_{(n)}$, имеет объемную плотность: $\mathbf{f}_{(n)}=\mathbf{f}_{(n n)}+\mathbf{f}_{(p n)}$. Здесь $\mathbf{f}_{(n)}=-\nabla x_{(n n)}$ - объемная плотность силы, действующей на каждую частицу $d B_{(n)}$ со стороны ее окружения в теле $B_{(n)}$, т.е. силы когезии $[20] ; \mathbf{f}_{(p n)}=-\nabla w_{(n p)}-$ объемная плотность силы, действующей на частицу $d B_{(n)}$ со стороны частиц $d B_{(p)}$, т. е. силы адгезии $[2,9,20]$.

6. Из всех возможных двух- и трехчастичных взаимодействий реализуются только те, которые можно описать на основании теории Ван-дер-ваальсовых сил и их флуктуаций $[11,16]$. Тогда в соответствии с результатами статистической механики твердого тела $[17,22]$ и с учетом групповых разложений Майера для малых объемов $d V_{i(n)} \approx \Delta V_{(n)}=1 / \beta_{(n n)}^{3}$ и малых значений отношений $\Delta U_{n n}^{(2)} / k T$ и $\Delta U_{n n n}^{(3)} / k T$ справедливо равенство

$$
\frac{\Delta U_{i(n) j(n) g(n)}^{(3)}}{k T}=\frac{\Delta U_{i(n) j(n)}^{(2)}}{k T} \frac{\Delta U_{j(n) g(n)}^{(2)}}{k T} \frac{\Delta U_{i(n) g(n)}^{(3)}}{k T} .
$$

Здесь $\quad \Delta U_{i(n) j(n)}^{(2)}=\Phi_{i(n) j(n)}^{(2)}\left(\Delta V_{(n)}\right)^{2}, \quad \Delta U_{i(n) j(n) g(n)}^{(3)}=$ $=\Phi_{i(n) 2(j) 3(g)}^{(3)}\left(\Delta V_{(n)}\right)^{3}$.

7. Взаимодействие частиц, принадлежащих двум телам из разных материалов, идентично взаимодействию тех же частиц в случае, когда они образуют частицу $\langle d B\rangle$ двухкомпонентного твердого раствора этих материалов [23] с объемными концентрациями компонент и соответственно. Модули Юнга $\langle E\rangle$ и сдвига $\langle G\rangle$, твердого раствора выражаются через модули его составляющих в соответствии с моделью Рейса - равенства нормальных и касательных напряжений в частицах $d B_{(n)}$ и $d B_{(p)}$, составляющих частицу $\langle d B\rangle$.

$$
\frac{1}{\langle E\rangle}=\frac{1-c}{E_{(n)}}+\frac{c}{E_{(p)}}, \quad \frac{1}{\langle G\rangle}=\frac{1-c}{G_{(n)}}+\frac{c}{G_{(p)}} .
$$

(Можно воспользоваться более строгими методами [24].)

8. Эффективная толщина поверхностного слоя любого из тел $B_{(n)}$, В котором концентрируется энергия его адгезии или поверхностная энергия при отсутствии адгезии $\left(h \approx 10^{-9} \mathrm{~m}[25,26]\right)$, много меньше характерных размеров сечения элементов биметаллического провода $\left(\approx 10^{-3} \mathrm{~m}\right)$, а также предлагаемой толщины промежуточного слоя $\left(\approx 10^{-6} \mathrm{~m}\right)$. Поэтому допускается, что взаимодействующие тела $B_{(n)}$ и $B_{(p)}$ полубесконечны, а поверхность их контакта $A_{(n p)}-$ плоскость. Приходящаяся на единицу площади плоскости $A_{(n p)}$ величина силы действия $F_{(n p)}$ такого тела $B_{(n)}$ на полубесконечное тело $B_{(p)}$ определяется выражением

$$
\begin{aligned}
& F_{(n p)}=\lim _{V_{(n)}, h(p) \rightarrow \infty} \int_{0}^{h(p)} d h_{(p)} \int_{V_{(n)}} f_{(n p)} d V_{(n)} \\
& \approx\left[\lim _{V_{(k)} \rightarrow \infty} \int_{V_{(2)}} f_{(k p)} d V_{(2)}\right]_{h(p)=0} .
\end{aligned}
$$

В (6) принято (по аналогии с [27]), что объемная плотность силы $F_{(n p)}$ распределена вблизи $A_{(n p)}$ равномерно в слое конечной толщины $h$. Это допущение позволяет с учетом результатов работ $[20,28]$ получить для вычисления $F_{(n p)}$ аналитическое выражение

$$
\begin{aligned}
& \frac{F_{(n p)}}{h}=\frac{105 \pi^{2}}{4}\left(\frac{\Phi_{0(p p n)}^{(3)}}{\beta_{(p p)}^{2} \beta_{(n p)}^{2}}\right)\left(\frac{1}{\beta_{(n p)}}-\frac{1}{\beta_{(p p)}}\right) \\
& +105 \pi^{2}\left(\frac{\Phi_{0(p n n)}^{(3)}}{\beta_{(n p)}^{5}}\right)-\left(\frac{7 \pi}{2}\right)\left(\frac{\Phi_{0(n p)}^{(2)}}{\beta_{(p n)}^{2}}\right) .
\end{aligned}
$$

Параметры $\beta_{(p p)}, \beta_{(n p)}, \Phi_{0(n p)}^{(2)}, \Phi_{0(p p n)}^{(3)}, \Phi_{0(p n n)}^{(3)}$, входящие в равенство (7), вычисляются с помощью следующих выражений, являющихся следствиями допущений $1-7$.

$$
\begin{gathered}
0.92 \cdot 10^{3}\left(\Phi_{0(p p)}^{(2)} / \beta_{(p p)}^{3}\right)=4 \mu_{(p)}+45 \lambda_{(p)}, \\
6.63\left(\Phi_{0(p p p)}^{(3)} / \beta_{(p p)}^{6}\right)=\lambda_{(p)}-\mu_{(p)},
\end{gathered}
$$

где $\lambda_{(p)}$ и $\mu_{(p)}$ - параметры Ламе материала с номером $(p)$.

$$
\beta_{(p p)}=\left[\frac{\left|\Phi_{0(p p)}^{(2)} / \beta_{(p p)}^{3}\right|^{3}}{(k T)^{2}\left|\Phi_{0(p p p)}^{(3)} / \beta_{(p p)}^{6}\right|}\right]^{1 / 6}
$$

где $k$ - постоянная Больцмана, $T-$ абсолютная температура. Формула (10) получена на основании равенства (4) для случая, когда расстоянием между частицами можно пренебречь. 
На основании допущений (4) и (7), учтенных при анализе потенциалов парных и тройных взаимодействий частиц твердого раствора, получены равенства

$$
\begin{gathered}
\left\langle\Phi_{0}^{(2)}\right\rangle=(1-c)^{2} \Phi_{0(11)}^{(2)}+2 c(1-c) \Phi_{0(12)}^{(2)}+c^{2} \Phi_{0(22)}^{(2)} \\
\left\langle\Phi_{0}^{(3)}\right\rangle=(1-c)^{3} \Phi_{0(111)}^{(3)}+3 c(1-c)^{2} \Phi_{0(112)}^{(3)} \\
+3 c^{2}(1-c) \Phi_{0(122)}^{(3)}+c^{3} \Phi_{0(222)}^{(3)} \\
\frac{4\langle\mu\rangle+45\langle\lambda\rangle}{0.92 \cdot 10^{3}}=\frac{\langle E\rangle}{0.92 \cdot 10^{3}} \frac{2+\langle v\rangle}{(1+\langle v\rangle)(1-2\langle v\rangle)} \\
=\left(\frac{\left\langle\Phi_{0}^{(2)}\right\rangle}{\langle\beta\rangle^{3}}\right)=(1-c)^{2}\left(\frac{\Phi_{0(11)}^{(2)}}{\beta_{(11)}^{3}}\right) \\
+2 c(1-c)\left(\frac{\Phi_{0(12)}^{(2)}}{\beta_{(12)}^{3}}\right)+c^{2}\left(\frac{\Phi_{0(22)}^{(2)}}{\beta_{(22)}^{3}}\right) \\
\frac{(\langle\lambda\rangle-\langle\mu\rangle)}{6.63}=\frac{\langle E\rangle}{13.26} \frac{4\langle v\rangle-1}{(1+\langle v\rangle)(1-2\langle v\rangle)}=\left(\frac{\left\langle\Phi_{0}^{(3)}\right\rangle}{\langle\beta\rangle^{6}}\right) \\
+3 c^{2}(1-c)\left(\frac{\Phi_{0(122)}^{(3)}}{\beta_{(12)}^{3} \beta_{(22)}^{3}}\right)+c^{3}\left(\frac{\Phi_{0(222)}^{(3)}}{\beta_{(22)}^{6}}\right) \\
=(1-c)^{3}\left(\frac{\Phi_{0(111)}^{(3)}}{\beta_{(11)}^{6}}\right)+3 c(1-c)^{2}\left(\frac{\Phi_{0(112)}^{(3)}}{\beta_{(11)}^{3} \beta_{(12)}^{3}}\right)
\end{gathered}
$$

Здесь $\langle v\rangle$ - коэффициент Пуассона твердого раствора. Параметр $\langle\beta\rangle$ определяется по формуле, структурно совпадающей с (10).

Допущения 1-8 и выражения (1)-(14) дают возможность вычислить значения параметров $\beta_{(p p)}, \beta_{(n p)}, \Phi_{0(n p)}^{(2)}$, $\Phi_{0(p p n)}^{(3)}, \Phi_{0(p n n)}^{(3)}$, характеризующих межчастичные потенциальные взаимодействия защитного материала слоя с материалом токопроводящей жилы и независимо от этого с материалом ее защитной оболочки и в конечном счете силу их взаимодействия $F_{(n p)}$.

Если при этом окажется, что для жилы (или оболочки) $F_{(n p)}<0$, то предполагаемый материал защитного слоя притягивается к жиле (или оболочке). В этом случае кандидатный материал надо заменить и повторить расчет для нового материала. Процесс необходимо повторять до тех пор, пока не будет выполнено неравенство $F_{(n p)}>0$. Оно означает отталкивание проверяемого материала защитного слоя материалом жилы (или оболочки). В этом случае для окончательного решения о выборе теоретически проверенного материала его защитные свойства необходимо проверить экспериментально. Очевидно, что конкретное значение эффективной толщины $h$ в этом случае не существенно.
Необходимо отметить, что указанным свойством должны обладать лишь те материальные волокна антиадгезионного слоя, которые ориентированы вдоль нормали к плоскости $A_{(n p)}$. В работах $[29,30]$ представлены результаты, позволяющие определить модули Юнга и сдвига материальных волокон слоистых материалов различной ориентации.

\section{Расчетное и экспериментальное подтверждения условия выбора материала антидиффузионного слоя}

Теоретическая и экспериментальная проверка представленного условия выбора материала защитного слоя, расположенного между медной токопроводящей жилой и стальной защитной оболочкой биметаллического провода, проведена для кандидатного материала талька, обладающего в направлении, перпендикулярном его слоям, близким к нулю значением коэффициента Пуассона.

В качестве исходных приняты следующие значения характеристик упругого состояния контактирующих материалов: материал $B_{(3)}-$ тальк $[31,32]$ : $E_{(3)}=0.16 \cdot 10^{11} \mathrm{~N} / \mathrm{m}^{2}, \quad v_{(3)}=0 ; \quad$ материал $B_{(1)}$ медь [5]: $E_{(1)}=1.10 \cdot 10^{11} \mathrm{~N} / \mathrm{m}^{2}, v_{(1)}=0.31$. Расчет модулей Юнга $\langle E\rangle$ и сдвига $\langle G\rangle$ проведен для концентрации $c=0.5$.

В результате оказывается, что $F_{(13)} / h=$ $=27.0 \cdot 10^{19} \mathrm{~N} / \mathrm{m}^{3}>0$. Значит, медная жила отталкивает защитный слой, адгезии между ними нет.

Аналогичный расчет проведен для случая, когда $B_{(2)}$ - сталь с характеристиками $E_{(2)}=2.12 \cdot 10^{11} \mathrm{~N} / \mathrm{m}^{2}$, $v_{(2)}=0.26$ [5]. При этом $F_{(23)} / h=174.4 \cdot 10^{19} \mathrm{~N} / \mathrm{m}^{3}>0$. Значит, стальная оболочка, как и медная жила, также отталкивает защитный слой из талька, и адгезии между ними нет.

Расчет, проведенный для оценки взаимодействия стали и меди в отсутствие антидиффузионного слоя, показал, что при их непосредственном контакте $F_{(12)} / h=-25.5 \cdot 10^{19} \mathrm{~N} / \mathrm{m}^{3}>0$. Значит, в отсутствие антидиффузионного слоя между медью и сталью возникает адгезия.

Таким образом, проведенный расчет подтвердил обоснованность теоретических положений, формирующих условие выбора материала антидиффузионного слоя.

В целях обеспечения экспериментального подтверждения теоретических положений по выбору материала антидиффузионного слоя была использована экспериментальная партия терможаростойкого биметаллического провода (рис. 1, $a, b)$, содержащего антидиффузионный слой, выполненный из талька, изготовленная совместно с ведущими российскими предприятиями. Образцы биметаллического провода подвергались термостарению в муфельной печи при температуре $550^{\circ} \mathrm{C}$ в течение $2500 \mathrm{~h}$. 

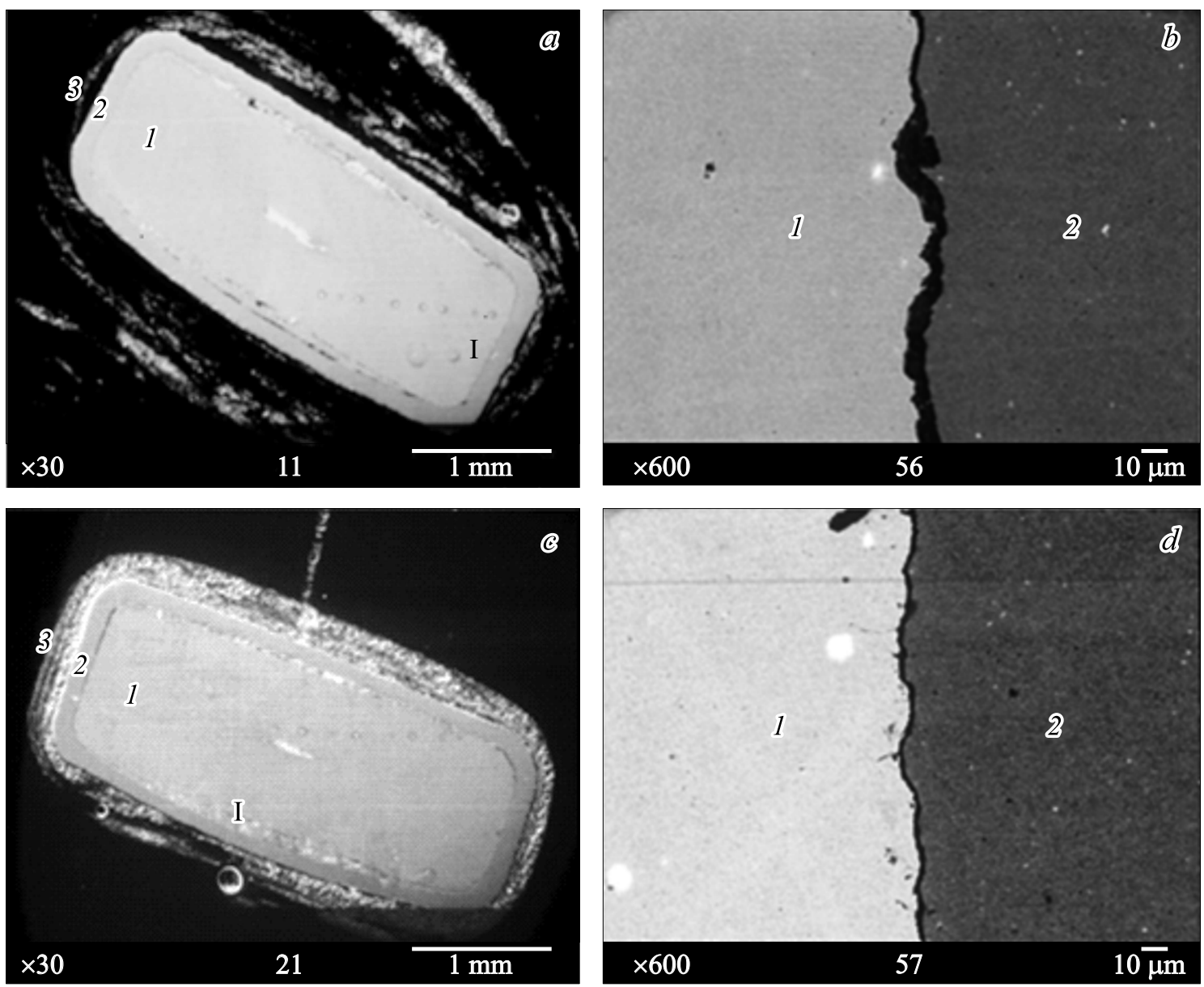

Рис. 1. Электронно-микроскопические снимки поперечного сечения терможаростойкого биметаллического провода до термостарения $(a, b)$ и после термостарения при $550^{\circ} \mathrm{C}$ в течение $2500 \mathrm{~h}(c, d): a, c-$ общий вид в спектре вторичных электронов, $b, d-$ $\mathrm{I}$ - участок в спектре отраженных электронов. 1 - основа, 2 - нержавеющая оболочка, 3 - изоляция.
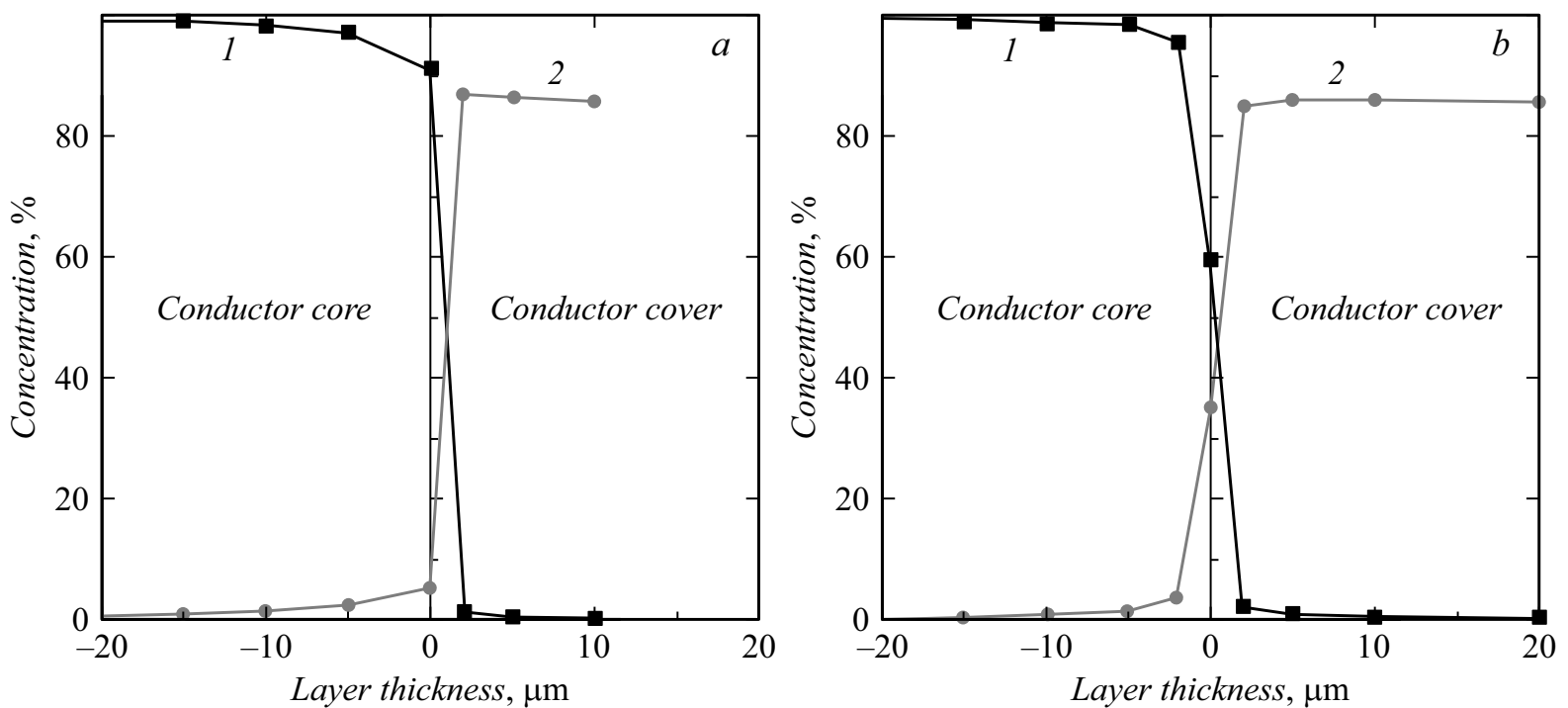

Рис. 2. Концентрационные профили меди (1) и железа (2) в образцах терможаростойкого биметаллического провода до (a) и после термостарения $(b)$. 
После термостарения методом электронной микроскопии выявлено, что взаимодиффузии материалов основы (1) и защитной оболочки (2) не произошло, так как границы контактных зон на изображениях электронной микроскопии четкие (рис. $1, c, d)$.

Отсутствие размытой границы на снимках и в результатах профилирования (рис. $2, a, b)$, полученных методом рентгеноструктурного микроанализа, свидетельствует о слабой интенсивности диффузионных процессов или их полном отсутствии. Наблюдаемые при этом переходные зоны не превышают $5 \mu \mathrm{m}$, что находится в пределах ошибки, связанной с локальным разрешением метода профилирования.

Таким образом, в условиях, интенсифицирующих процессы диффузии, экспериментально показано, что материал, обладающий антиадгезионными свойствами по отношению к паре материалов, между которыми возможна адгезия, препятствует и их взаимной диффузии. Проведенные исследования использованы при подготовке патента на изобретение [33].

\section{Заключение}

Представленное в работе теоретическое построение условия выбора материала антидиффузионного слоя между составляющими терможаростойкого биметаллического обмоточного провода, защищающего их от взаимной диффузии, его расчетное и экспериментальное подтверждение, дает основание полагать, что это условие может быть использовано в качестве критерия теоретической оценки защитных возможностей кандидатных антидиффузионных материалов по отношению не только к традиционно используемым в электротехнической промышленности токопроводящим материалам, но и новым материалам специального назначения.

Разработанная методика выбора материала антидиффузионного слоя, выполненная на основе теоретического построения, с проработанной базой данных позволит существенно сократить временные и материальные ресурсы при создании многослойных систем заданного назначения.

Работа выполнялась в порядке личной инициативы и в рамках базовой части государственного задания шифр 1.5265.2017/БЧ на 2017-2019 гг.

\section{Список литературы}

[1] Vitkovsky I.V., Golovanov M.M., Kirillov I.R. et al. // Russian Conference on Magnetohydrodynamics. June 22-25, 2015. Perm. P. 111.

[2] Физический энциклопедический словарь / Гл. ред. Б.А. Введенский. М.: Сов. энциклопедия, 1960. T. 1. C. 19.

[3] Магомедов М.Н. Изучение межатомного взаимодействия, образования вакансий и самодиффузии в кристаллах. М.: Физматлит, 2010. 540 с.
[4] Драпкин Б.М., Руденко В.А. // ЖТФ. 1992. Т. 62. Вып. 9. C. $125-130$.

[5] Андреев Т.В. и др. Свойства элементов / Под ред. Г.В. Самсонова. Ч. 1. Физические свойства. 2-е изд., перераб. и доп. М.: Металлургия, 1976. 599 с.

[6] Горелик С.С., Дашевский М.Я. Материаловедение полупроводников и диэлектриков. М.: МИСИС, 2003. 480 с.

[7] Дехтяр И.Я. // УФН. 1957. Т. LXII. Вып. 2. С. 99-128.

[8] Партенский М.Б. // УФН. 1979. Т. 128. Вып. 1. С. 69-106.

[9] Лифиии E.M. / Труды Е.М. Лифшица / Под ред. Л.П. Питаевского, Ю.Г. Рудого. М.: Физматлит, 2004. 646 с.

[10] Дерягин Б.В., Кротова Н.А. // УФН. 1948. Т. XXXVI. Вып. 3. С. 387-406.

[11] Бараш Ю.С. Силы Ван-дер-Ваальса. М.: Наука, 1988. $344 \mathrm{c}$.

[12] Tersoff J. // Phys. Rev. B. 1988. Vol. 37. N 12. P. 6991-7000.

[13] Huang H., Ghniem N.M., Wong J.K., Baskes M.I. // Modeling Simul. Mater. Sci. Eng. 1995. N 3. P. 615-627.

[14] Ruelle D. Statistical mechanics: Rigorous results. N.Y., Amsterdam: V.A. Benjamin, Inc., 1969. 219 p.

[15] Литинский Г.Б. // Вісник Харківского національного університету. Хімія. 2010. Вып. 18(4). № 895. С. 26-30.

[16] Ландау Л.Д., Лифшии Е.М. Теоретическая физика. Квантовая механика (нерелятивистская теория). М.: Наука, 1989. T. $3.768 \mathrm{c}$.

[17] Майер Дж., Гепперт-Майер М. Статистическая механика. М.: Мир, 1980. 544 с.

[18] Векилов Ю.Х., Вернгер В.Д., Самсонова М.Б. // УФН. 1987. Т. 151. Вып. 2. С. $341-376$.

[19] Шоркин В.С. // Известия РАН МТТ. 2011. № 6. C. $104-121$.

[20] Шоркин В.С., Фроленкова Л.Ю., Азаров А.С. // Материаловедение. 2011. № 2. С. 2-7.

[21] Romashin S.N., Presnetsova V.Yu., Frolenkova L.Yu., Shorkin V.S. // Advanced Structured Materials. 2016. Vol. 1. N 42. P. 363-376.

[22] Жирифалько Л. Статистическая физика твердого тела. М.: Мир, 1975. 384 c.

[23] Dolgov N.A., Romashin S.N., Frolenkova L.Yu., Shorkin V.S. // Nanomechanics and Technology: An International J. 2015. Vol. 6. N 2. P. 117-133.

[24] Горбачев В.И., Емельянов А.Н. // Известия РАН МТТ. 2014. № 1. С. $95-107$.

[25] Лейббрид Г. Микроскопическая теория механических и тепловых свойств кристаллов. М., Л.: Физматгиз, 1963. $312 \mathrm{c}$.

[26] Киселев В.Ф., Козлов С.Н., Зотеев А.В. Основы физики поверхности твердого тела. М.: МГУ, 1999. 384 с.

[27] Горячева И.Г., Маховская Ю.Ю. // ПММ. 2001. Т. 65. Вып. 2. С. 279-289.

[28] Фроленкова Л.Ю., Шоркин В.С. // Вестник Пермского национального исследовательского политехнического ун-та. Механика. 2013. № 1. С. 235-259.

[29] Гольдштейн Р.В., Городиов В.А., Лисовенко Д.С. // Физическая мезомеханика. 2014. Т. 17. № 5. С. 5-14.

[30] Гольдштейн Р.В., Городиов В.А., Лисовенко Д.С. // ДАН. Механика. 2009. Т. 429. № 5. С. 614-616.

[31] Вахромеев Г.С., Ерофеев Л.Я., Канайкин В.С., Номоконова Г.Г. Петрофизика: учебник для вузов. Томск: Изд-во Том. ун-та, 1997. $462 \mathrm{c.}$

[32] Ерофеев Л.Я., Вахромеев С.С., Зинченко В.С., Номоконова Г.Г. Физика горных пород: учебник для вузов. Томск: Изд-во Том. ун-та, 2006. 520 с.

[33] Патент РФ № 2611054. 27.08.2015. Белослудцев К.Н., Витковский И.В. Провод обмоточный терможаростойкий. Бюл. № 6. Опубл. 21.02.2017. 